\title{
Prinzip und Stabilität von Locking-Platten in der Traumatologie des Gesichtsschädels
}

\author{
Ralf Gutwald, Marc Metzger, Rainer Schmelzeisen, Vincent Coupez
}

\section{Zusammenfassung}

Ein neues internes Mini-Locking-System wurde im Vergleich zu herkömmlichen 2,0-mm-Miniplatten getestet. Standardisierte Osteotomien im Unterkieferwinkel bei 16 menschlichen Leichen wurden mit einer 6-Loch-Platte an der Linea obliqua fixiert. Die Osteosynthese und die Stabilität der Verankerung wurden mit einem 3-dimensionalen In-vitro-Modell, in dem eine funktionale Belastung simuliert wurde, geprüft. Der Vergleich der verschiedenen Methoden der Osteosynthese zeigte, dass bei der Fixierung mit Miniplatten eine vermehrte Verdrehung (Torsion) und Distanzierung der Knochenfragmente während des Anziehens der Schrauben auftraten, wenn die Platten auf den Knochen angepresst wurden. Es werden letzte Inkongruenzen von Platten- zu Knochenoberfläche auf die mobilen Knochenfragmente übertragen, was zu vermehrten Verdrehungen und Distanzierung führt. Dies wurde zu einem geringeren Ausmaß mit dem Mini-Locking-System beobachtet, weil das Fixierungsprinzip den Druck auf den Knochen vermeidet. Bei funktioneller Belastung zeigte das Mini-Locking-System auch eine signifikante höhere Stabilität im Vergleich zu herkömmlichen Miniplatten. Durch das Fixierungsverfahren, das die Prinzipien des Fixateurs imitiert, bilden die Schrauben zusammen mit der Platte und dem kortikalen Knochen eine Rahmenkonstruktion. Belastungskräfte werden ohne Plattenreibung direkt vom Knochen über die Schraube auf die Platte übertragen, was zu einer höheren Stabilität führt.

\section{Principle and Stability of Locking Plates in the Treatment of Injuries to Facial Bones}

A new internal mini-locking system was tested and compared with conventional $2.0 \mathrm{~mm}$ miniplates. Standardised osteotomies in the angular region of 16 human cadaver mandibles were fixed with a 6-hole plate at the oblique line. Osteosynthesis and stability of fixation were proven in a three-dimensional in-vitro model in which a functional load was simulated. Comparison of the different osteosynthesis techniques showed that, in the case of miniplate fixation, torsion and gapping of the bone fragments occurred following plate application and screw tightening when the plates were pressed onto the bone, so lasting incongruences between bone surface and plate were transferred to the mobile bone fragments, thus resulting in more extended gaps and torsion. This was only observed to a much lesser extent with the mini-locking system due to the fixation principle which avoids pressure to the bone. During functional loading the mini-locking system showed also a significantly higher stability in comparison to conventional miniplates. Due to the fixation method imitating the principles of a fixateur, the screws form a frame construction together with the plate and the cortical bone. Loading forces are transmitted without the need of plate friction directly from the bone over the screws to the plate resulting in a higher stability.

\section{Einleitung}

Mit der Entwicklung der Osteosynthese in der Mund-, Kiefer- und Gesichtschirurgie wurden verschiedene Systeme entwickelt, die kleiner, einfacher zu handhaben geworden sind und extraorale Verfahren vermeiden. Mittlerweile hat sich die Fixierung von Unterkieferfrakturen mit Miniplatten zu einer Standardbehandlung entwickelt [1]. Dennoch wurden Komplikationen bei der Miniplatten-Osteosynthese des Unterkiefers v.a. im Kieferwinkelbereich in bis

OP-JOURNAL 2012; 28: 184-187

(c) Georg Thieme Verlag KG Stuttgart · New York DOI http://dx.doi.org/10.1055/s-0032-1315374 zu 30\% der Fälle beschrieben. Hierzu gehören die Bewegung und das Lösen von Schrauben, die zum Scheitern der Frakturbehandlung führen. Außerdem stört der Druck der Platte die Blutversorgung, was zu Knochennekrose im Bereich der Schrauben und zum Verlust der Friktion der Platte führt.

Um die Miniplatten-Osteosynthese zu verbessern, wurde ein neues internes Mini-Locking-System in Zusammenarbeit mit dem AO/ASIF-Institut (Davos, Schweiz) entwickelt.

\section{Materialien und Methoden}

Dimensionen des neuen Mini-LockingSystems sind mit herkömmlichen 2,0-
mm-Miniplatten-Systemen vergleichbar. Zusätzlich hat die selbstschneidende 2,0mm-Locking-Schraube ein spezielles doppeltes Gewinde um den Schraubenkopf.

Die Locking-Platte hat ein entsprechendes Innengewinde in den Schraubenlöchern. Beim Einsetzen verriegelt sich die Locking-Schraube in das korrespondierende Gewinde der Plattenlöcher (Abb. 1).

In einer präklinischen Untersuchung am AO/ASIF-Institut (Davos, Schweiz) haben wir die Handhabung und mechanische Stabilität des neuen Mini-Locking-Systems gegenüber dem herkömmlichen 2,0-mm-Miniplatten-System bewiesen. 


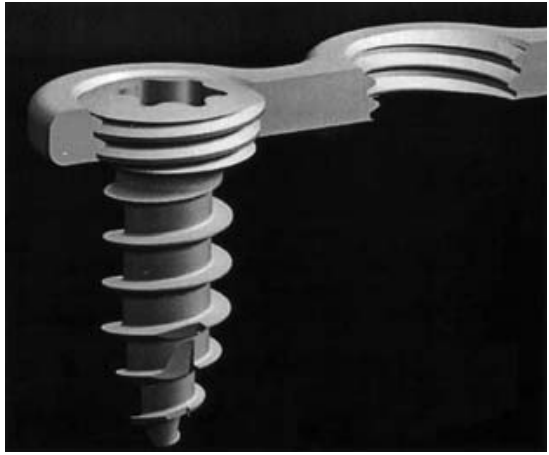

Abb. 1 Mini-Locking-System (Plattendicke $1,0 \mathrm{~mm}$, Schraube Außendurchmesser 2,0 mm).

Standardisierte Osteotomien im rechten Unterkieferwinkel wurden bei 16 menschlichen Leichen durchgeführt und mit einer 6-Loch-Platte (Länge $40 \mathrm{~mm}$, Dicke $1,0 \mathrm{~mm}$ ) und 6 Schrauben (Länge $6,0 \mathrm{~mm}$, Außendurchmesser $2,0 \mathrm{~mm})$ eines jeden Systems an der Linea obliqua fixiert.

Acht Unterkiefer wurden mit dem MiniLocking-System und 8 Unterkiefer mit konventionellen Miniplatten fixiert.

Um den Einfluss der anatomischen Unterschiede auszuschalten, wurden die Unterkiefer in 2 äquivalente Testgruppen nach der Matched-Pairs-Methode aufgeteilt.

Nach der Osteosynthese wurden die Spalten (Distanzierung) und die Torsion an den Osteotomiespalten (kranial und kaudal) gemessen.

Die mechanische Stabilität der verschiedenen Fixationsmethoden wurde gemäß der Kroon-Methode [3] mit einem 3-dimensionalen In-vitro-Modell (Abb. 2) getestet.

Die Anwendung von funktionellen Belastungen mit 20, 35, 50 und $65 \mathrm{~N}$ wurde an 9 verschiedenen Stellen entlang der Zahnreihe durchgeführt. Die funktionalen Belastungen wurden nacheinander ohne Anziehen der Schrauben bei jeder Belastung durchgeführt. Änderungen der Dimension als Reaktion auf Distraktion oder Kompression wurden computergestützt an 3 Stellen der Osteotomie (kranial, lingual, kaudal) mit Dehnungsmessstreifen erfasst.

Statistische Auswertungen wurden mit Varianzanalysen durchgeführt (General mixed model analysis of variance, Mann-Whitney-Test).

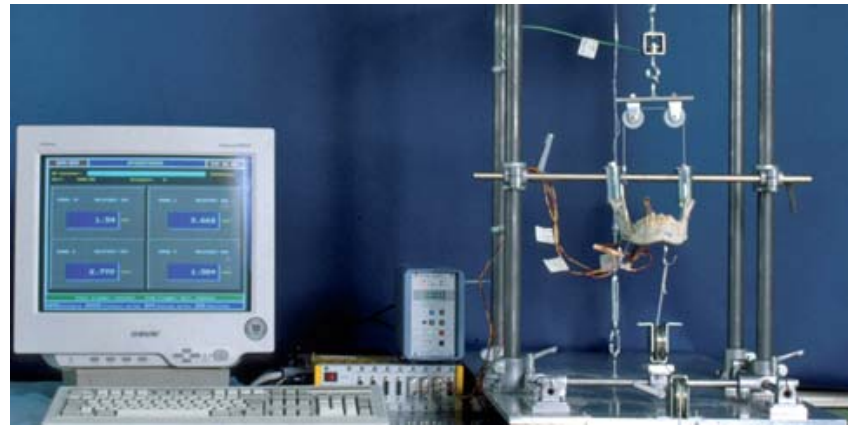

Abb. 2 3-D-Belastungs-In-vitro-Modell.

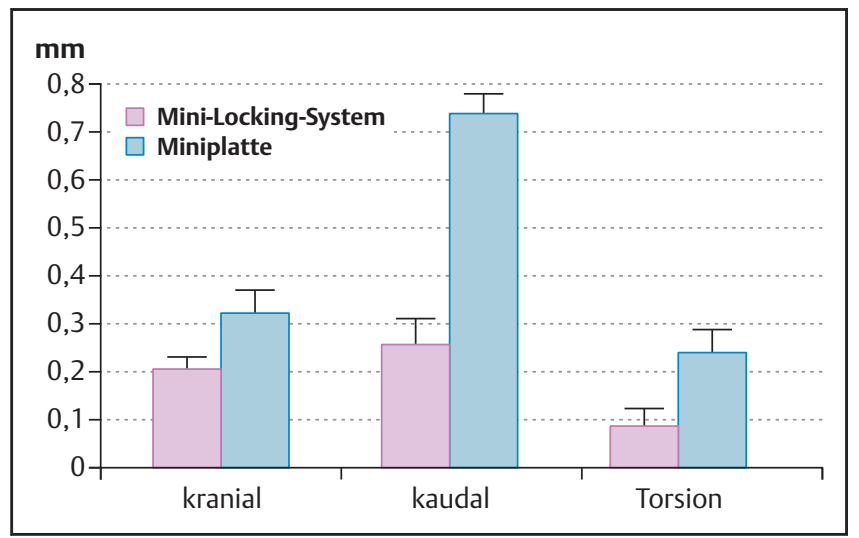

Abb. 3 Distanzierung und Torsion nach Osteosynthese.

\section{Ergebnisse}

Nach der Osteosynthese zeigten die Unterkiefer, die mit dem Mini-Locking-System versorgt wurden, die kleinste Spaltmasse (Distanzierung) und Torsionen.

Im Vergleich zu den Miniplatten waren die Unterschiede signifikant (Abb. 3). An der kaudalen Stelle der Osteotomie waren sie am signifikantesten $(\mathrm{p}=0,010)$.

Bei der Simulation der Muskelkräfte mit mechanischer Belastung wurden in beiden Gruppen die typischen zunehmenden Dislokationen am kaudalen Rand des Unterkiefers beobachtet, wenn die Belastung näher an die Osteotomiestelle übertragen wurde (Abb. 4).

Außer den zunehmenden Dislokationen wurden keine Unterschiede bez. der Charakteristik der Verteilung von Werten beim Erhöhen der funktionellen Belastung beobachtet.

Im Vergleich zu den verschiedenen Methoden der Fixierung zeigte das MiniLocking-System die beste Stabilität.

In Bezug auf die 4 verschiedenen Belastungskräfte waren die Werte in allen Belastungspositionen signifikant niedriger für das Mini-Locking-System als für die

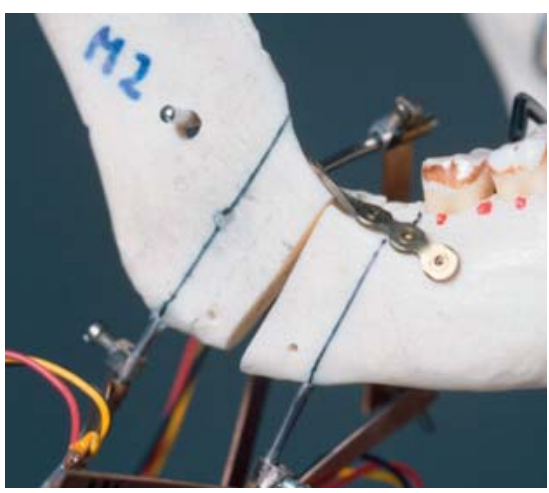

Abb. 4 Osteotomie am Kieferwinkel mit Mini-Locking-System fixiert während der funktionellen Belastung. Typische Dislokation am kaudalen Rand des Unterkiefers, wenn die Belastung näher an die Osteotomiestelle übertragen wurde.

Miniplatten. Die höchste Signifikanz $(p=0,015)$ zeigte sich am kaudalen Dehnungsmessstreifen (Abb. 5).

\section{Diskussion}

Der Vergleich der verschiedenen Methoden der Osteosynthese zeigte, dass bei der Fixierung mit Miniplatten vermehrte Verdrehung (Torsion) und Distanzierung der Knochenfragmente während des Anziehens der Schrauben auftraten, wenn die Platten auf den Knochen angepresst wurden. 
Dies ist das Ergebnis der unterschiedlichen Fixierungsmethode. Bei Verwendung konventioneller Miniplatten ist es essenziell, die Platte exakt an die Knochenoberfläche zu adaptieren. Es werden letzte Inkongruenzen von Plattenzu Knochenoberfläche beim Anziehen der Schrauben auf die mobilen Knochenfragmente übertragen. Damit kommt es zu vermehrter Verdrehung und Distanzierung und einem primären Repositionsverlust.

Wenn das Mini-Locking-System mit Locking-Schrauben fixert wird, bleibt die Reposition nahezu unverändert. Daher muss die Platte nicht so genau wie bei konventionellen Plattensystemen adaptiert werden.

Bei der mechanischen Belastung wurden in beiden Gruppen die typischen zunehmenden Dislokationen am kaudalen Rand des Unterkiefers beobachtet, wenn die Belastung näher an die Osteotomiestelle übertragen wurde $[3,4]$.

Andererseits zeigte das Mini-LockingSystem die beste Stabilität. Dies ist auch das Ergebnis der unterschiedlichen Fixierungsmethode.

Bei der konventionellen Technik drückt das Anziehen der Schrauben die Platte gegen den Knochen. Dieser Druck erzeugt Friktion, die wesentlich zur Primärstabilität beiträgt (Abb.6). Am Schraubengewinde hat nur die Seite, die zu der Platte orientiert ist, an der Entwicklung des Druckes auf die Platte Anteil.

Bei dem Mini-Locking-System werden die auftretenden Kräfte vom Knochen direkt auf die Schrauben, von dort auf die Platte und auf der anderen Frakturseite wieder über die Schrauben auf den Knochen übertragen. Die Reibung zwischen Platte und Knochen ist zur Stabilität nicht notwendig. Auf jeder Frakturseite verankern sich die Schrauben zum einen in der Platte und zum anderen in der äußeren Kortikalis, sodass ein mechanisch starrer stabiler Rahmen gebildet wird (Abb. 7). Kräfte an der Schraube werden besser über die ganze Gewindekontur verteilt.

In Trümmer- oder Defektfrakturen kann die Fixierung mit konventionellen Plattensystemen zur sekundären Dislokation führen, wenn der Druck zwischen Platte und Knochen nicht mehr gewährleistet ist. Die Plattenosteosynthese mit Locking-Schrauben kann diese Art von

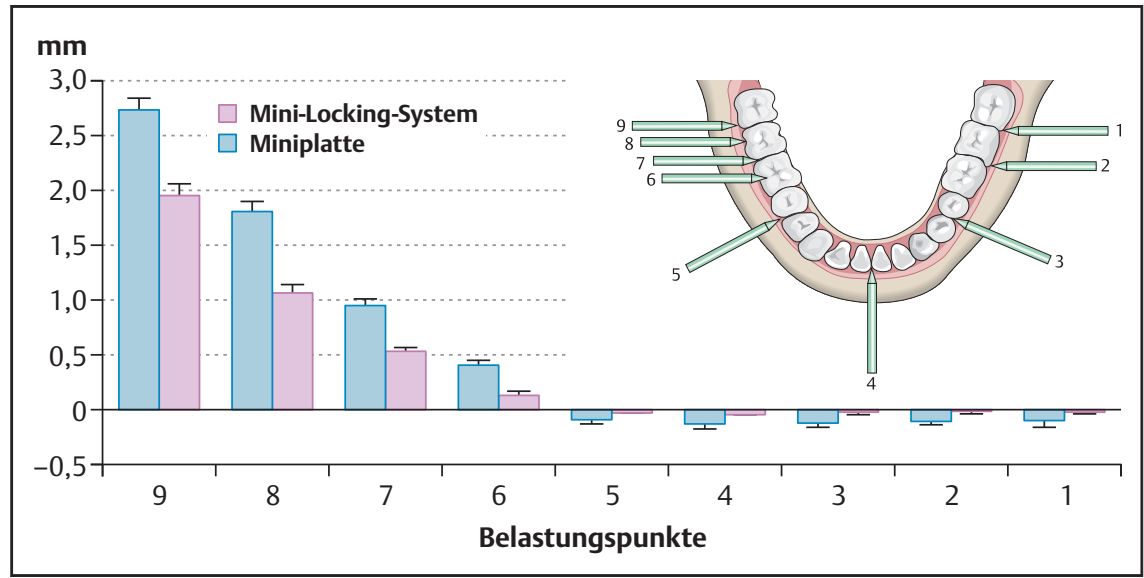

Abb.5 Distraktion und Kompression an der kaudalen Seite der Osteotomie (Belastung mit 35 N). Rosa Säulen Mini-Locking-System. Hellblaue Säulen Miniplatte.

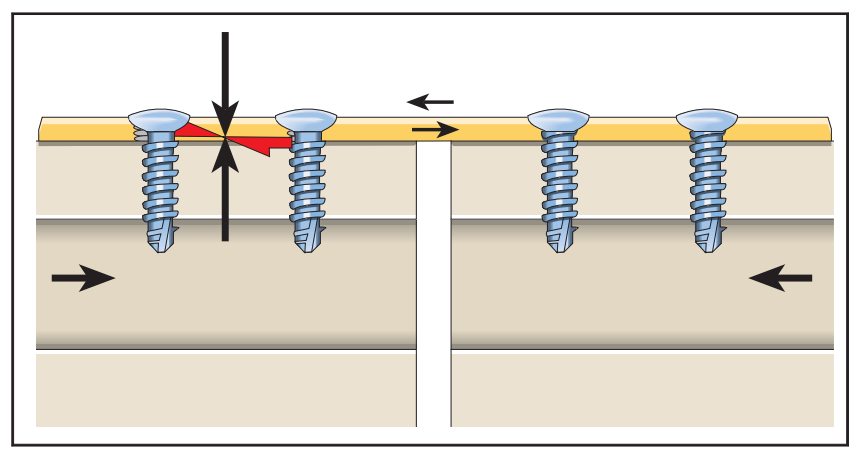

Abb. 6 Primärstabilität bei konventioneller Miniplatte. Pfeil = Belastungsverteilung und Druck/ Gegendruck (Reibung) an der Platte/ Knochen.

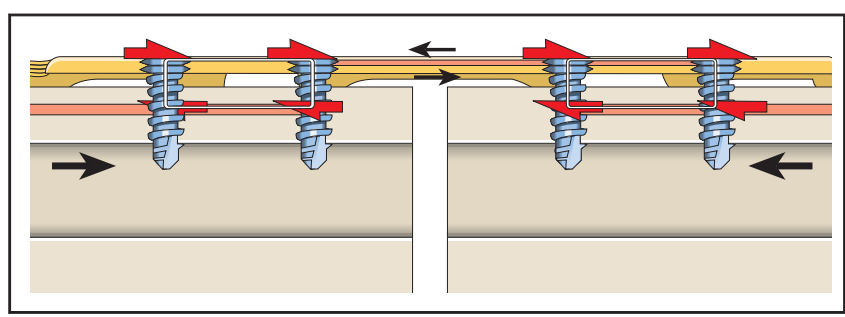

Abb. 7 Primärstabilität beim Mini-Locking-System. Pfeil = Belastungsverteilung direkte Übertragung vom Knochen auf die Schrauben, von dort auf die Platte und auf der anderen Frakturseite wieder über die Schrauben auf den Knochen). Rechtecke = mechanisch starrer Rahmen als Ergebnis der Verriegelung der Schraube in der Platte sowie in den Knochen.

sekundärer Dislokation vermeiden. Auch in schlechter Knochenqualität kann die Verankerung der Schrauben zu einer Lockerung und damit zum anschließenden Verlust der Reposition führen. Durch die sichere Verriegelung der Schraube in der Platte kann dieses Problem mit dem MiniLocking-System vermieden werden.

\section{Schlussfolgerung}

Das neue interne Mini-Locking-System kombiniert 2 Prinzipien mit mehreren Vorteilen.
Das Locking-Prinzip verhindert das Abziehen sowie die Bewegung und die Lockerung von Schrauben. Die Fixierungstechnik imitiert die Prinzipien eines Fixateurs.

Dies vereinfacht die Biegung der Platte und vermindert die Torsion oder die Distanzierung an der Frakturstelle. Die erhöhte Stabilität ist das Ergebnis des mechanisch starren Rahmens und der Belastungsübertragung wie oben beschrieben. Der fehlende Druck unter der Platte verhindert Interferenzen mit der Gefäß- 

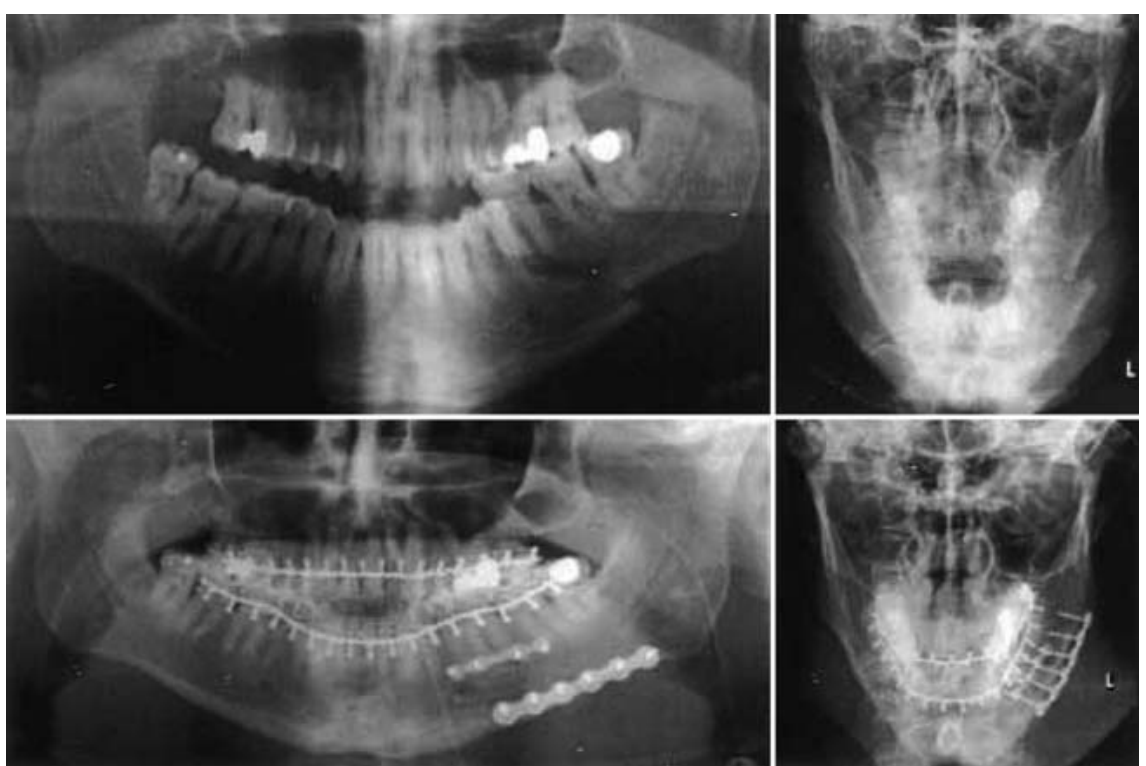

Abb. 8 Prä- und postoperative Röntgenaufnahmen einer schweren dislozierten Fraktur, Corpus mandibula links. Fixation mit 2 Mini-Locking-Platten (klein und medium).

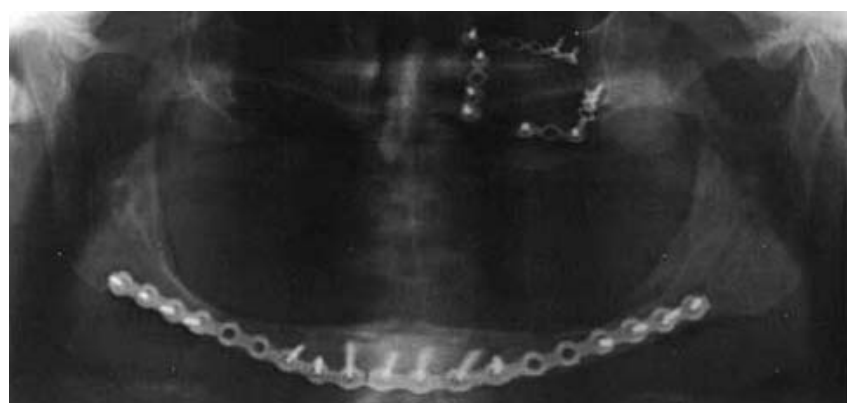

Abb. 9 Postoperative Röntgenaufnahme einer doppelseitigen Fraktur eines atrophischen Unterkiefers. Fixation mit 2 MiniLocking-Platten (medium); zusätzlich eine linke Jochbogenfraktur mit 3 Mini-Locking-Platten fixiert (klein).

versorgung des Knochens und ermöglicht das Periostwachstum unter den Platten, was die Frakturheilung unterstützt. Dies wurde klinisch bei allen mit Mini-Locking-Platten behandelten Frakturen bei der Plattenentfernung nach 6 Monaten beobachtet.

Zusätzlich zu den kleinen Mini-LockingPlatten wurden 2 größere Platten für ein breiteres Spektrum von Indikationen entwickelt. Die 2,0-mm-Schraube passt $\mathrm{zu}$ allen Plattenkonfigurationen und kann mono- oder bikortikal angewendet werden. Bei Bedarf können auch Non-
Die ersten klinischen Anwendungen mit allen 3 Plattenprofilen zeigten hervorragende Ergebnisse. Besonders bei schweren Frakturen und Trümmerfrakturen (Abb. 8) sowie bei Frakturen des atrophischen Unterkiefers (Abb. 9) ist die Osteosynthese mit dem Mini-Locking-System mittlerweile eine Standardtherapie in unserer Klinik geworden.

\section{Literatur}

${ }^{1}$ Schmelzeisen R, McIff T, Rahn B. Further development of titanium miniplate fixation for mandibular fractures. Experience gained and questions raised from a prospective clinical pilot study with $2.0 \mathrm{~mm}$ fixation plates. J Craniomaxillofac Surg 1992; 20: 251-256

${ }^{2}$ Ellis 3rd E, Walker L. Treatment of mandibular angle fractures using two noncompression miniplates. J Oral Maxillofac Surg 1994; 52: 1032-1036

${ }^{3}$ Kroon FH, Mathisson M, Cordey JR et al. The use of miniplates in mandibular fractures. An in vitro study. J Craniomaxillofac Surg 1991; 19: 199-204

${ }^{4}$ Schierle HP, Schmelzeisen R, Rahn B et al. Oneor two-plate fixation of mandibular angle fractures? J Craniomaxillofac Surg 1997; 25: 162-168

Prof. Dr. med. Dr. med. dent. Ralf Gutwald

Leitender Oberarzt

Priv.-Doz. Dr. med. Dr. med. dent.

Marc Metzger

Oberarzt

Prof. Dr. med. Dr. med. dent.

Rainer Schmelzeisen

Ärztlicher Direktor

Dr. med. dent. Vincent Coupez

Assistenzarzt Locking-Schrauben, die eine größere Angulation erlauben, benutzt werden.
Klinik für Mund-Kiefer-

Gesichtschirurgie

Uniklinik Freiburg

Hugstetter Straße 55

79106 Freiburg

vincent.coupez@uniklinik-freiburg.de 